\title{
Discurso pronunciado por el doctor Pedro Nel Cardona, Presidente del V Congreso Colombiano de Obstetricia y Ginecología, durante su sesión inaugural.
}

\section{Señor Gobernador del Departamento,}

Señores Presidentes Honorarios del Congreso de Obstetricia y Ginecología,

Señor Rector de la Universidad de Antioquia,

Señor Presidente de la Academia de Medicina de Medellín,

Señor Decano de la Facultad de Medicina,

Señor Médico Jefe del I.C.S.S.,

Señor Presidente de la Asociación Antioqueña de Obstetricia y Ginecología,

Señoras y señores:

Es para mí honroso y grato, presentar a ustedes en nombre de la Asociación Antioqueña de Obstetricia y Ginecología, y en el mío, un efusivo saludo de bienvenida a Medellín, ciudad acordada hace cuatro años como sede oficial de las sesiones ordinarias del V Congreso de nuestra especialidad.

Circunstancias especiales, suficientemente conocidas, retardaron por dos años la reunión del actual Congreso, lo cual ha contribuído a reforzar el entusiasmo que hoy contemplamos, al iniciar las presentes jornadas, que prometen ser torneos de la inteligencia, más que simples reuniones protocolarias, y en donde espero flamearán ideas renovadoras, revaluación de conceptos pretéritos, confirmación de principios, recordatorio de conocimientos en trance de olvido, recreación del espíritu médico y euforia del entendimiento.

El Congreso Latinoamericano reunido en Bogotá a mediados del año pasado, patrocinado por la FLASOG y organizado por la Sociedad Coiombiana de Obstetritica y Ginecología tuvo un éxito magnífico. Sea esta la oportunidad, aunque tardía, para hacer llegar a sus organizadores, aquí presentes, nuestro voto de aplauso y felicitación, por el bello certamen realizado, modelo de organización y dechado de cultura, con asistencia de los más altos exponentes de la obstetricia y ginecología internacionales. 
Los congresos médicos se han generalizado a todos los ámbitos de la ciencia de Hipócrates. No es, pues, extraño que nosotros, amantes del bienestar social de la mujer, nos reunamos a parlamentar acerca de sus problemas patológicos, con el fin de conocerlos más en sus modalidades regionales y concatenarlos en forma regular, con miras a la unidad médica nacional, enantes desarticulada, como nuestra misma geografía patria, con aparente unidad civil pero con extensas regiones a donde escasamente llega el eco trémulo que la brisa susurra sobre el pabellón tricolor del Capitolio Nacional.

Aquí están presentes nuestros fraternos colegas de la Costa Atlántica, que vienen a traernos observaciones regionales, problemas desconocidos en el territorio limítrofe de las vecinas repúblicas australes, y a su vez, tendremos también ocasión de conocer corrientes que desde Pasto y Popayán confluyen a este hermoso Valle del Aburrá, y así, en una comunión de ideas, podamos conocernos más, vincularnos más, amarnos más, enseñarnos los unos a los otros, toda vez que en el imperio de la ciencia médica no existen castas ni privilegios distintos a la verdad, único objetivo de nuestra existencia, y que la Providencia reparte por igual a todos los mortales, a condición del esfuerzo personal, como el que nos tiene congregados aquí, con miras a nuestro perfeccionamiento profesional y científico.

Si se me permite el símil, en este momento nos encontramos en el Sancta Sanctorum de la Obstetricia y Ginecología nacionales, con ánimo erguido al sacrificio de nuestros intereses personales, en bien de la comunidad, sin más deseos de recompensa que la satisfacción espiritual que sintieron — entre otros- Pasteur, Newton o Galileo, cuando depositaron su ofrenda en el altar sacrosanto de la eterna sabiduría.

Sin embargo, no debemos engañarnos con la idea errónea de que la asistencia a estos congresos equivale a la de un cursillo de información médica, en donde se desarrolla un temario acordado previamente y dictado por profesores universitarios, mediante conferencias académicas y prácticas hospitalarias. Los que tal cosa pensaren, se sentirían completamente defraudados, porque aquí no asistimos a las aulas de los viejos claustros docentes sino que, por el contrtario, nos reunimos a parlamentar, como en las cámaras de los países democráticos, con libertad absoluta de expresión, libertad de apreciación de los conceptos, libertad de cultos en el terreno científico y libertad en la revelación de las experiencias personales. Más que a aprender detalles interesantes, venimos a refinar conocimientos y a purificar ideas. 
Existe un consenso universal favorable a los congresos. El fervor aumenta año por año, como lo demuestra la nutrida asistencia, entre otros, al de Obstetricia y Ginecología de Viena en 1961, y al de Moscú, sobre Cancerología, en 1962. Uno de los reporteros de una revista norteamericana se dio a la tarea de inquirir, a los asistentes del Congreso moscovita, la impresión personal que se habían formado acerca de la magna reunión. Tan sólo el $8 \%$ se consideraron defraudados, en mi concepto, por razones baladíes, tales como el costo excesivo del viaje o cuestiones idiomáticas, pero ninguno hizo protesta de inutilidad propiamente dicha o inconveniencias de otro orden.

El programa general del congreso es más práctico que especulativo. Los temas de relato fueron acordados en la sesión de clausura de nuestra última reunión en la ciudad de Barranquilla. La comisión organizadora se permitió hacer algunas variaciones accidentales tendientes a la vinculación de todas las secciones del país, sin exclusivismos ni preferencias tendenciosas. Tanto la obstetricia como la ginecología serán apreciadas por igual y con idéntico interés.

Vamos a conocer guarismos estadísticos de nuestra patología nacional que serán de gran utilidad para las entidades oficiales y aún para nuestras Facultades de Medicina. En Antioquia, por ejemplo, la frecuencia del prolapso genital es algo extraordinario; más de la mitad de la cirugía que se hace en la Cátedra de Ginecología de nuestra Facultad es referente a pacientes con tal patología. En Inglaterra, tradicionalmente ha sido también muy frecuente esta entidad ginecológica; en cambio, en algunas regiones alemanas, es muy escasa, casi excepcional. Nos decía el profesor de Ginecología de la Universidad de Stöingen, ciudad del sur, que en su servicio era una rareza el hallazgo de un prolapso genital y era motivo de especiales comentarios cuando se presentaba un caso en su clínica hospitalaria.

Sabemos que el prolapso genital es enfermedad exclusiva del tejido conjuntivo, pero hay algo - tal vez nutricional - que no conocemos todavía y que estamos en mora de investigar, lo cual esperamos será motivo de una comunicación el próximo Congreso, si nuestras pesquisas cristalizan en algo positivo. Otro capítulo muy interesante que se ventilará en esta ocasión, es el referente a mola y corion, entre nosotros. Parece que en la región del Quindío se presenta la entidad con caracteres alarmantes, aún mayores que en las Filipinas y China Comunista, en donde según informes de la doctora Acosta Simson, registran el índice más alto conocido en el mundo. La ponencia sobre este tema, tanto el relato como el correlato, nos prometen dar números estadísticos de importancia trascendental.

Quiero destacar que en esta ocasión se hizo invitación especialísima a todas y cada una de las asociaciones obstetro-ginecológicas del país, para que las 
conclusiones que podamos presentar, sean el refiejo fiel de nuestra patología nacional. Por primera vez, en la historia de estos Congresos, oiremos relatos procedentes de Cartagena y Cúcuta que nos llenan de satisfacción.

El advenimiento, pudiéramos llamar, de la endocrinología al terreno ginecológico, ha transformado radicalmente su programa. La patología funcional del ovario, entrelazada con las disfunciones hipotalámicas, hipofisiarias, tiroidianas y córtico-suprarrenales, ha alcanzado tal preponderancia, que no sería exagerado afirmar que más o menos la mitad de las consultas ginecológicas giran por la órbita de la endocrinología.

En la hora actual miramos como un anacronismo al médico de antaño, cuando trataba de hallar en el examen clínico la lesión orgánica explicativa de los trastornos femeninos, fundamento del problema en cuestión.

Cuántas metrorragias funcionales, trastornos del desarrollo corpora1, flujos inespecíficos, dismenorreas rebeldes, trastornos del ciclo menstrual, mastopatías funcionales, aberraciones múltiples inexplicables, abortos seriados, trastornos mentales, síndromes adreno-genitales, el mismo Chiari-Frommel, el síndrome de Cushing y muchos más estados patológicos que sería largo enumerar, tienen - todos- un substrato ovárico, en estrecha relación con sus glándulas satélities, y que el ginecólogo moderno está en la obligación de conocer, estudiar, investigar, diagnosticar y tratar convenientemente.

Y pasando al campo somático, nos encontramos con lesiones tangibles, tales como la endometriosis externa, el fíbroma uterino, la adenomiosis, el Stein-Leventhal, quizá el mismo prolapso genital, algunos tumores glandulares de la mama, y aún el enigmático carcinoma genital, casi todos ellos estados patológicos, en los cuales encontramos su etiología ubicada en la zona endocrinológica. De lo dicho podemos deducir, que el ginecólogo actual es - por derecho propioun verdadero endocrinólogo, un científico de alcurnia y un clínico sagaz, que está llamado a afrontar los más complicados problemas de la patología femenina, en armonía con los progresos de la ciencia y marchando hombro a hombro con sus avanzadas de honor.

El ginecólogo dejó de ser el modesto médico de tiempos pasados, tratante de cervicitis gonocócicas, metroanexitis neisserianas y trastorns del ciclo. Hoy en día, sin descuidar aquellos sencillos menesteres de otros tiempos, afronta problemas muy complicados de psiquiatría, endocrinología, citología exfoliativa, de radiaciones actínicas, anatomía patológica, medicina preventiva, laboratorios médicos y técnicas quirúrgicas modernas. 
Es, pues, la ginecología una especialidad por lo alto en contraposición a la de las primeras décadas del presente siglo. Sin embargo, es de justicia señalar que el avance de la ginecología exige elementos de trabajo, sin los cuales es imposible madurar los diagnósticos, ni cosechar los tratamientos respectivos. Sirva de ejemplo - entre nosotros - el de una paciente con adenoma córtico-suprarrenal, en sus comienzos. El cuadro clínico es confuso, y el diagnóstico diferencial es igualmente difícil. Pero, con la colaboración de los rayos $\mathrm{X}$, el estudio de los esteroides urinarios, las pruebas de estimualción y depresión con ACTH y cortisona respectivamente, y una buena neumopelvimetría, se llega a un diagnóstico preciso. Es verdad que la mayoría de los casos no son tan complejos como el mencionado, pero sí es necesario disponer siquiera de laboratorios de anatomía patológica, de citología exfoliativa, de rayos $\mathrm{X}$, y de un buen laboratorio clínico para salir avante en el ejercicio de la ginecología moderna.

Otro punto cardinal en el ejercicio del ginecólogo es el relativo al carcinoma genital. En los dos primeros Congresos nacionales realizados en Bogotá y Cali, se presentaron algunos trabajos relacionados con el carcinoma del cérvix uterino. Se pensó entonces que el tema había sido agotado, y se excluyó del programa en las reuniones de Ibagué y Barranquilla. Hoy abogo por su inclusión en ios próximos congresos, como tema extraordinario en importancia científica y social. El solo hecho de su frecuencia en nuestro país sería más que justificación para dedicarle toda nuestra atención y nuestro esfuerzo. Se sabe que de cuatro personas, una hará un cáncer inevitablemente. Su localización es imposible predecirla. Pero si dirijimos la mirada a la mujer, es el cáncer uterino el sitio de su predilección. En manos del ginecólogo está el problema y su solución.

El desarrollo de la lucha contra el cáncer en un país, es directamente proporcional a su economía, dice Karnofsky en reciente publicación. El enunciado es aceptable con alguna salvedad: En el terreno investigativo, estamos de acuerdo; pero en lo referente a profilaxis y métodos de tratamiento, es imposible cruzarse de brazos en espera de una economía mejor.

En realidad, el porcentaje de morbilidad, por tal concepto, es hoy más o menos lo mismo de hace 20 años y no obstante, sus índices de mortalidad son menores. Con medidas adecuadas, en Rusia, v. gr., en el año de 1949, el diagnóstico oportuno se hacía únicamente en el $44 \%$, y en la actualidad, alcanza ya el $63.7 \%$. El número de casos avanzados, en el mismo país, ha disminuído de $43 \%$ en 1949 , a $21.3 \%$ en 1960 .

Con un esfuerzo más, a pesar de nuestra ignorancia en su etiología y patogenia, el hombre eliminará definitivamente la enfermedad cancerosa de localiza- 
ción uterina. Ojalá corramos igual suerte con el carcinoma ovárico, hoy por hoy enigmático en sus comienzos, y de una gravedad intrínseca incomparable.

La psiquiatría como ciencia que trata de las enfermedades mentales, ha llegado - a última hora - al campo de la ginecología con ímpetu incontenible. En sí misma, la psiquiatría es tan vieja como el hombre, pero su estudio metódico, acorde con las manifestaciones dolorosas y funcionales en el campo ginecológico, es un nuevo capítulo, llamado a ser de importancia inmensa en nuestras clínicas.

Hace ya casi un lustro tenemos implantada esta sección en el Servicio Hospitalario de San Vicente de Paúl y cada día podemos apreciar más y más sus benéficos resultados. Fue justamente el motivo que nos inspiró a modificar el programa general acordado en Barranquilla, al introducir un relato sobre el particular, el cual, con su correlato correspondiente, será discutido en las actuales sesiones. Juzgo necesario subrayar ante ustedes la importancia de la psiquiatría ginecológica, porque con su adpoción se cambia fundamentalmente la conducta terapéutica en la mayoría de los casos.

Yo siempre me pregunto: ¿Cuántas drogas, especialmente antibióticos, analgésicos y tranquilizantes, se prescriben innecesariamente? ¿Cuántos regímenes agobiadores, mal balanceados, se aconsejan con rumbo incierto? ¿Cuántos tratamientos hormonales, aunque bien intencionados, desacertados en esencia? ¿Cuánta cirugía inútil o aún perjudicial, en busca de lesiones fantasmas? En mi opinión que todos estos errores se pueden evitar si el ginecólogo medita un poco en estos cuadros psícopáticos, y piensa dos veces la terapéutica a seguir en presencia de aquel crecido número de pacientes, sedientas de cirugía o de tratamientos hormonales.

Tan importante como la ginecología, y quizás más importante aún, es la obstetricia, ciencia básica de la felicidad y el bienestar de la sociedad humana. La medicina estudia al enfermo, y el médico se hace -como quien dice- responsable de una vida. La obstetricia, en cambio, se responsabiliza con dos vidas a la vez. El obstetra en ejericico, es un profesional de mérito excepcional, es casi un semidiós, como tal lo aprecia la sociedad y por eso le rinde culto de admiración y respeto. Los problemas que presenta la obstetricia entre nosotros son muchísimos y de gran trascendencia. Para su acertada solución se hace necesario una legislación especial por parte del Estado, y una preparación científica de los profesionales dedicados a tal fin, que haga de ellos verdaderos especialistas, ojalá - siquiera en los grandes centros urbanos- de dedicación exclusiva, para mejor provecho de la comunidad. Nos tocó en suerte vivir en el siglo de las grandes realizaciones, y la obstetricia debe enfilar dentro de ellas. 
El estudio consciente y metódico de la paciente en el curso del embarazo, es de una importancia inapreciable. Pero no se trata de un examen superficial, con metro en mano, midiendo la altura uterina, y prescribiendo rutinariamente un específico a base de vitaminas. Esa modesta labor, claro está, podría estar al alcance de cualquier enfermera de escasa preparación universitaria. Mas, lo que yo entiendo por consulta prenatal, es cosa totalmente diferente: Es un estudio clínico completo, de acuerdo con cada caso particular, en donde alternan la clínica propiamente dicha y todos sus complementos indispensables como radiografías, pelvimetría, exámenes microscópicos de flujos, citología exfoliativa funcional, dosificaciones hormonales, exámenes completos de orina y sangre, lo mismo que los cropológicos y demás que se consideren necesarios.

Esta labor es parte integrante del médico dedicado a la obstetricia y no improvisada al acaso; debe ejercerse con responsabilidad, con entusiasmo, con amor; dándose cuenta de su importancia y sabiendo que no sólo es meritoria en sí, sino la gran profilaxis de las complicaciones del parto. Con esta consulta prenatal se lograría reducir al mínimo: a) el temor infundado a la maternidad; b) gran número de abortos, cuando obedecen a problemas hormonales, nutricionales, psicologicos, o de otra patología tratable en oportunidad; c) muchas toxicosis gravídicas, con cuadros de albuminuria, hipertensión, preclampsia y estados afines; d) las hemorragias de la gestación en general, especialmente las que se refieren a defectos de coagulación, afibrinogenesis, etc.; e) algunas aplicaciones inconvenientes de fórceps, por ejemplo, las que tienen lugar en el estrecho superior, por falta de estudio previo; f) igualmente algunos desgarros uterinos en el curso del parto; g) gran parte de la mortalidad perinatal, y h) de una vez por todas la implantación del método psico-profiláctico, benéfico a todas luces, y que resulta inhumano desconocerlo por desidia, o abandonarlo por negligencia y falta de voluntad o prevención.

Me abstengo de analizar —uno por uno- los apartes mencionados, por razones de espacio; pero tengo la seguridad de que la mente de mis oyentes es terreno abonado por la comprensión y el patriotismo.

Aunque brevemente, me voy a permitir decir unas pocas palabras en relación con el parto mismo, que es por naturaleza el epicentro de todas las consideraciones obstétricas. En primer término nos encontramos con el máximo problema del parto, o sea el dolor. Es consenso universal que el parto duele y duele mucho. Desde el precepto bíblico, a través de todas las edades, y de generación en generación, el parto ha sido doloroso. Filosóficamente, y más aún, fisiológicamente, no debiera serlo, porque se trata de un órgano normal, el útero, en trance de cumplir una función biológica, dentro de las leyes naturales de la misma biología. No es igual pero sí muy semejante a las funciones normales que cumplen, por ejemplo, el estómago, el intestino o la vejiga urinaria, al expulsar 
su conenido, sin el más mínimo dolor. Hay sensación, pero no molesta y aflictiva que son las características del dolor. Pero señores, ante la realidad de los hechos no hay argumento posible, el parto sigue siendo doloroso y muy doloroso, a pesar de todo.

Ahora bien, si mediante las recomendaciones aconsejadas por el método psicoprofiláctico, se logra un alto porcentaje de sedación y alivio, en semejante trance, pregunto yo: ¿será humanitario dejar en nuestro país las cosas como están? ¿Será acaso necesario que los poderes públicos impongan la medida bajo severas sanciones? ¿No sería más meritorio, conveniente, útil y hasta elegante, que las clínicas de maternidad oficiales y semioficiales, como el Seguro Social, abocaran de lleno el problema, sin más dilaciones sofísticas ni evasivas de cualquier otro orden? Es sabido por todos nosotros que en los EE. UU., en Rusia, y en la mayor parte de los países europeos el método está ya establecido y marcha normalmente. En Colombia misma, se ha implantado ya en algunas clínicas particulares de Bogotá, Barranquilla, Bucaramanga y otras ciudades; pero mi pensamiento es más amplio, y ambicioso, pues lo que deseo es que se generalice por todos los ámbitos del país, empezando, naturalmente, por las clínicas universitarias.

Los variados problemas inherentes a la clínica del parto, ya sea normal o distócico, no son tema abordable por mí en esta ocasión, cuando solamente he tratado de referirme a ideas generales, y en ningún caso a los pormenores y detalles de técnica o sus complicaciones, así sean ellos, a veces, altamente interesantes. Hago, pues, gracia a cualquier reflexión referente a las aplicaciones de fórceps, operación cesárea, procidencias del cordón, feto muerto retenido, desgarros perineales, embarazo con carcinoma genital, hemorragias graves en el curso del parto, traumatismos uterinos, asepsia y antisepsia en obstetricia, problemas de anestesia, etc., pero sí quiero llamar la atención al estudio cuidadoso de la mortalidad perinatal, que para bien de las presentes jornadas, será motivo de amplia discusión, como uno de los temas de más alto significado. En el curso del debate esperamos oír conceptos sobre su profilaxis y números estadísticos que nos ilustren sobre el particular.

En cuanto a tradición, quiero recordar que, a poca distancia de estos muros, en el corazón mismo del Valle de Aburrá, rival en belleza del paraíso que nos describe el Génesis en sus primeras páginas, fue donde se practicó, con buen éxito, la primera operación cesárea, en los tiempos aquellos extraordinarios de los albores de la república. El cirujano fue el doctor José Ignacio Quevedo, precursor genial de nuestra cirugía, oriundo de Cundinamarca y médico que fue del general Francisco de Paula Santander. Por derecho propio, podemos pues, llevar vara de palio, en los anales de la obstetricia y ginecología nacionales. 
No tenemos por qué extrañarnos de la multiplicidad de problemas tendidos sobre la mesa. Creo yo que andamos mejor nosotros, ginecólogos y obstetras, que otros profesionales ajenos a la medicina, en donde encontramos un verdadero caos, por ejemplo en las finanzas nacionales, administración pública, la impunidad en la rama judicial y tantos otros puntos álgidos de la actualidad del país, que en verdad flaquean las fuerzas de la nación, conturban el ánimo y amilanan los espíritus.

A pesar de todo, yo comparto con el expresidente Lleras la fe inquebrantable en Colombia. Este país no puede ser un engendro frustrado del genio de Bolívar. Tenemos una tradición de grandeza, y una herencia idiomática y religiosa que no alcanzaríamos a malgastar en muchos lustros. Cada uno de nosotros es potencialmente capaz de neutralizar la acidez corrosiva de los enemigos de la civilización cristiana, en la proporción de uno por diez mil.

Carísimos congresistas: vuestra asistencia a estas reuniones redundará naturalmente en provecho propio, porque aquí se ventilarán problemas de ambas especialidades que a todos interesan; por mi parte hago votos sinceros para que así sea. Pero también es de justicia recordaros que además estais haciendo un sacrificio económico que sería innoble menospreciar. Nos damos perfecta cuenta de lo que representa para cada uno el abandono del trabajo por varios días, con las erogaciones mayores y menores inevitables.

Además, y ésto es lo más meritorio, la colaboración científica que habeis aportado, con trabajos personales saturados de experiencia, tanto en obstetricia como en ginecología, y que tendremos ocasión de conocer en el curso de las presentes sesiones.

Si sacrificios desinteresados por el bien común se suelen premiar con el simbólico laurel, todos vosotros os habeis hecho acreedores al galardón, y podeis estar seguros de que la patria anotará vuestros nombres en el libro de oro, que la conciencia pública se encarga de guardar con gran cuidado, para estímulo de muchos y honor de vuestros descendientes.

Por el éxito de este Congreso, de índole exclusivamente nacional, empinemos enhiestos el espíritu, y desde el fondo del alma exclamemos SURSUM CORDA, para que el avance de la obstetricia y la ginecología entre nosotros sea progresivo e indefinido, y muy especialmente para que la ETICA profesional, aquel preciado conjunto de normas y virtudes inmutables, que modelan al caballero y engrandecen al médico, sea el escudo luminoso, distintivo de todas nuestras actividades. 